\title{
Feline Alveolar Osteitis: Osseodensification with Implant Placement Protocol
}

\author{
Rocco E Mele ${ }^{1}$ and Gregori M Kurtzman ${ }^{2 *}$ \\ ${ }^{1}$ VCA Valley Animal Hospital and Emergency Center, Tucson, AZ, USA \\ ${ }^{2}$ Silver Spring, MD, USA
}

*Corresponding author: Gregori M Kurtzman, General practitioner, International dental author and lecturer, Leisure World Plaza Professional Building, 3801 International Drive, Suite 102, Silver Spring, MD 20906, USA, Tel: 301-598-3500; Email: drimplants@aol.com

\section{Case Report \\ Volume 6 Issue 2}

Received Date: April 05, 2021

Published Date: May 04, 2021

DOI: $10.23880 /$ oajvsr-16000212

\section{Abstract}

Feline dental implants are becoming a predictable and viable treatment option for the replacement of lost canines due to maxillary alveolar osteitis (AO) a painful condition, commonly experienced by felines. Surgical extraction and debridement remains the treatment of choice for this complex inflammatory process. Future complications however can be a common sequela of maxillary canine loss. The case presented demonstrates successful surgical extraction of a maxillary canine with implant placement following the osseodensification protocol and utilizing the sockets osteitis buttressing bone formation to promote a positive result with final restoration of the implant with a crown 13 weeks post implant placement.

Keywords: Alveolar Osteitis; Osseodensification; Implant; Tooth Resorption

\section{Introduction}

Alveolar osteitis $(\mathrm{AO})$ is a chronic inflammatory process more frequently diagnosed in maxillary canine sockets of the feline patient. The clinical presentation of AO may include oral pain, bleeding, periodontitis, tooth resorption (ORL), and alveolar buccal bone changes [1-5].

A presumptive diagnosis clinically of $\mathrm{AO}$ is made on the awake patient, documenting clinical features such as; gingivitis with soft tissue swelling, gingival mucosal erythema, buccal bone expansion, and coronal extrusion (Figure 1). Radiographically, changes are identified under general anesthesia. Those bony changes and pathology may include; deep palatal probing (Figure 2 red), alveolar bone expansion (Figure 2 green), buttressing condensing bone
(Figure 2 blue) and a mottled osseous appearance mimicking rough, large trabeculae (Figure 2 yellow).

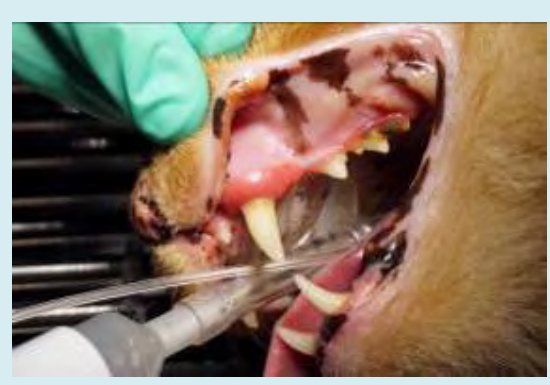

Figure 1: Alveolar Osteitis (AO) associated with the maxillary left canine. 


\section{Open Access Journal of Veterinary Science \& Research}

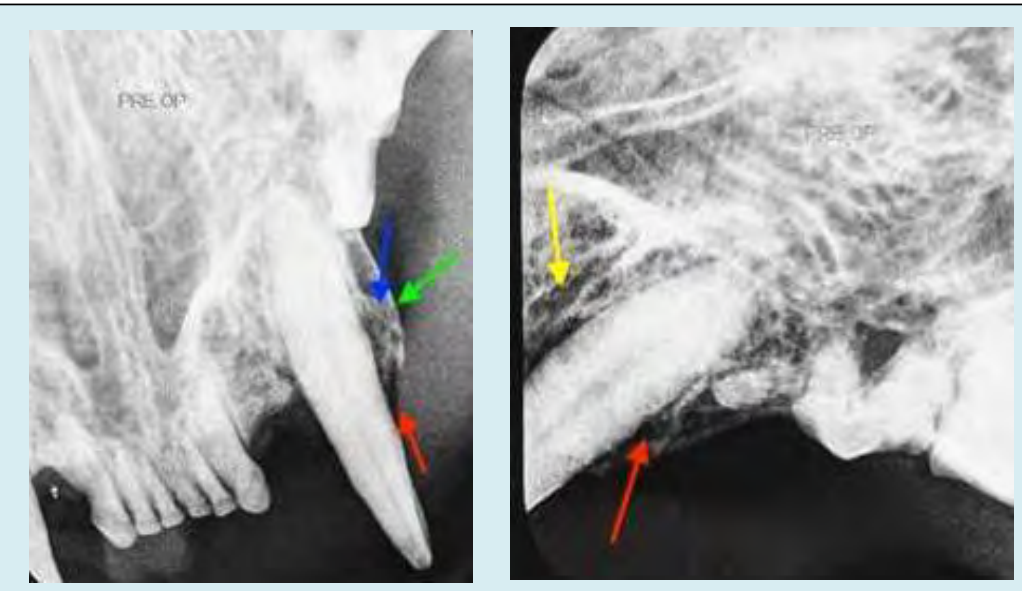

Figure 2: Deep probing (red) with osseous expansion of the socket wall (green) and condensing bone (blue) demonstrating a mottled trabeculae pattern in the radiograph (yellow).

\section{Osseodensification}

Osseodensificaiton is a novel biomechanical bone preparation technique for dental implant placement to improve bone quality by increasing its density utilizing Densah burs (Versah, Jackson, MI). The burs are rotated in a counterclockwise direction (reverse mode) at $800-1500$ rpm. While standard osteotomy drills remove and excavate bone during implant site preparation, the Densah burs allow bone preservation by condensation through compaction. This results in autografting during osteotomy preparation thereby increasing the peri-implant bone density (\% BV) and the resulting implants mechanical stability [6].

Osseous densification has demonstrated an increase in the percentage of bone at the implant surface by increasing the bone mineral density in the peri-implant region. Bone compaction has been reported in numerous studies to improve early implant fixation and better load handling through increased bone density at the bone implant interface [7-9].

\section{Case Presentation}

A 9 year old neutered male DLH feline weighing $5 \mathrm{~kg}$ presented for evaluation of "swollen gums". Initial oral examination noted: alveolar bone expansion with tooth extrusion (\#204) with gingival erythema and mild tooth mobility (M1) (Figure 1). A preliminary diagnosis of alveolar osteitis was made with a final diagnosis and treatment plan to be presented to the owner at the time of general anesthesia following a complete oral exam.

\section{Surgical Phase}

Blood was drawn the morning of the scheduled oral surgical procedure for a complete blood count (CBC) and diagnostic profile to evaluate the patient's general health. Results were all in the normal range.

Patient was premedicated with atropine sulphate (MWI Veterinary Supply, Boise, Idaho) $0.01 \mathrm{mg} / \mathrm{kg}$ subcutaneously (SQ) and Acepromazine Maleate (MWI Veterinary Supply) $0.02 \mathrm{mg} / \mathrm{kg} \mathrm{SQ}$. An intravenous catheter was placed and lactated ringers solution was started at a rate of $3 \mathrm{ml} / \mathrm{kg} / \mathrm{h}$. General anesthesia was induced by mask with Sevoflurane (Sevo) (MWI Veterinary Supply). The Sevo Vaporizer was set at \#7 and $\mathrm{O}_{2}$ flow was set at $1 \mathrm{~L}$. Intubation with a cuffed endotracheal tube was completed, and the anesthetic agent was maintained at the vaporizer setting of $3 \% . \mathrm{O}_{2}$ flow rate was set at $1 \mathrm{~L} / \mathrm{M}$. A unilateral left maxillary infraorbital nerve block was administered with $0.5 \%$ Bupivacaine (Benco Dental, Tucson, AZ) at $0.1 \mathrm{ml}$ at the site and Buprenorphine (MWI Veterinary Supply) at $0.01 \mathrm{mg} / \mathrm{kg}$ IV following the General Anesthetic Protocol

A complete oral examination and digital PA radiographs were obtained and a final diagnosis of alveolar osteitis was made. The owner was notified of the results and the treatment options available which included: 1) Surgical extraction (XSS) with socket debridement. 2) XSS with particulate allograft. 3) XSS with possible immediate implant placement. The feline's owner chose to place an immediate implant, if possible and a future restoration following implant healing.

A sulcular incision was made to create an envelope flap with a distal vertical releasing incision for a full thickness flap to expose the underlying bone and to allow atraumatic removal of the affected canine (\#204) and maximize maintenance of the surrounding bone and tissue vascularity making future implant/restoration more predictable (Figure 3). Socket debridement was performed following extraction of the affected canine utilizing small curettes and copious sterile saline lavage with removal of any connective tissue, 


\section{Open Access Journal of Veterinary Science \& Research}

bacterial contaminants and root/bone fragments from the socket ensuring a clean interface between the implant and alveolar bone.

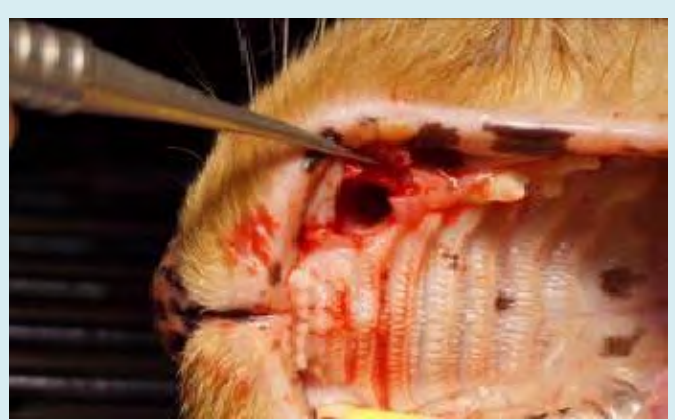

Figure 3: Following extraction of the affected canine a full thickness envelop flap is elevated to expose the buccal aspect of the extraction socket.

Socket osteotomy was accomplished with an osseodensification drilling protocol referred to as compaction auto-grafting utilizing Densah Burs. The burs and technique compress the osteotomized alveolar bone increasing its density to yield better primary stability and superior boneto-implant contact when the implant is inserted (Figure 4). The bur geometry rotating counter-clockwise (reverse mode) at a rotary speed of 800 to $1500 \mathrm{rpm}$ with profuse saline irrigation prevents bone over-heating, while allowing compaction of the bone along the inner surface of the implant osteotomy without osseous cutting. A gentle bouncing motion (in and out movement) is helpful to create a ratedependent stress to produce a rate-dependent strain and allowing saline solution pumping to gently pressurize the boney walls. This combination facilitates an increased bone plasticity and resulting bone expansion [10-13].

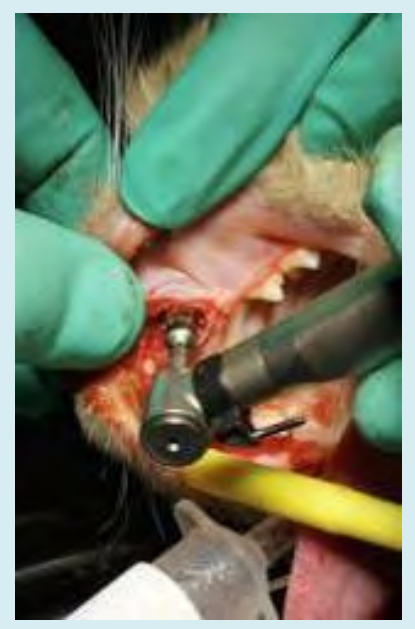

Figure 4: Densah bur is utilized in the extraction socket to condense the osteotomy improving the bone density (quality) that will house the implant.
A $5.0 \mathrm{~mm} \times 8.0 \mathrm{~mm}$ engage endosseuos implant (OCO Biomedical, Albuqerque, NM) was secured to a $4 / 5 \mathrm{~mm}$ internal hex driver and attached to a Mont Blanc 20:1 implant handpiece. The implant motor (Aseptico, Woodinville, WA) was set at $45 \mathrm{Ncm}$ with a speed of $20 \mathrm{rpm}$. The implant was introduced into the condensed site to a depth of $4 / 5 \mathrm{~mm}$ subcrestal at which point the handpiece torqued out at $45 \mathrm{Ncm}$ (Figure 5). The evolution of Implant Stability Quotient (ISQ) values to access implant secondary stability demonstrated statistically significant correlation with implant outcome. In fact, no implant with $>60$ failed, while $19 \%$ of implants ISQ $<60$ failed [14]. The implant was tested for Implant Stability Quotient (ISQ) using the Osstell IDX unit (Osstell USA, Baltimore, MD) read 65.

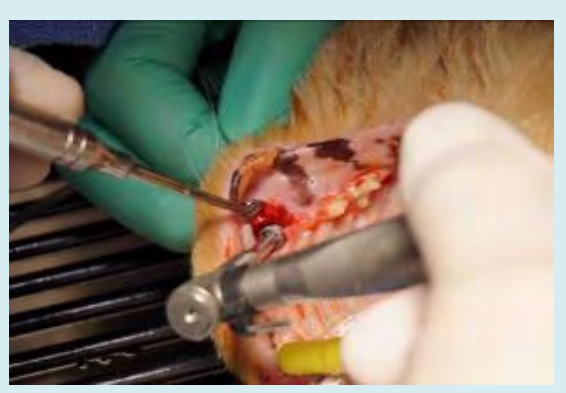

Figure 5: Implant placement is performed utilizing the surgical handpiece into the prepared osteotomy.

A cover screw was inserted into the implant to prevent graft material from entering the implants connection at the platform. A condenser was utilized to pack an allograft (Veterinary Transplant Service (VTS), Kent, WA) into the gap between the implant and socket walls at the crest. The graft material not only fills the "jump gap" between the implant and bone but occupies the tissue zone to the height of the free gingival margin (FGM). The graft material is incorporated into the tissue zone, acting as a scaffold to support the ridge contour profile and peri-implant tissue [15] (Figure 6). The cover screw is carefully removed, so as not to disturb the graft and replaced with a $4 / 5 \mathrm{~mm}$ flat healing abutment (OCO Biomedical) with a height slightly taller than the thickness of the soft tissue so that a single stage surgical approach could be utilized. This acts as a prosthetic seal and is tightened with finger pressure utlizing a hand held hex driver. The flap margins were reapproximated around the healing abutment and fixated with Securocryl, a 5/0 synthetic suture material (Securos Surgical, Fiskdale, MA) in an interrupted manner (Figure 7). Post-Op radiographs were taken to evaluate the implant/abutment interface and graft placement (Figure 8) Recovery was uneventful and the patient was discharged with post-surgical instructions to the animal's owner on the same day as surgery. Cefovecin Sodium was administered $\mathrm{SQ}$ at a dose of $0.045 \mathrm{ml} / \mathrm{lb}$ (Zoetis, Inc., Kalamazoo, MI) and 
Buprenorphine (MWI Veterinary Supply, Boise, ID) at a dose of $0.01 \mathrm{mg} / \mathrm{kg}$ orally every 12 hours for 5 days.

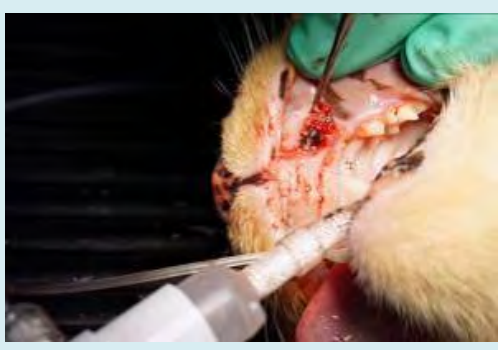

Figure 6: Osseous graft material is placed into the gap between the implant and wall of the extraction socket prior to flap closure.

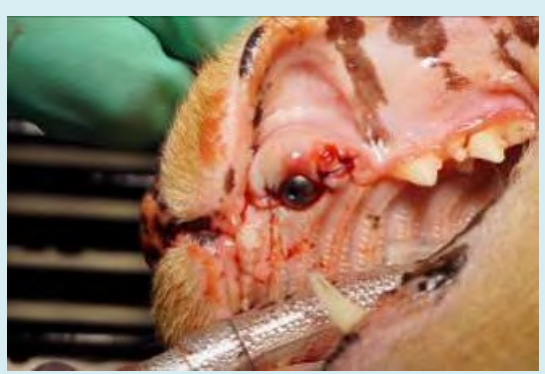

Figure 7: The soft tissue flap is approximated to achieve primary closure around the healing abutment and secured with interrupted sutures.

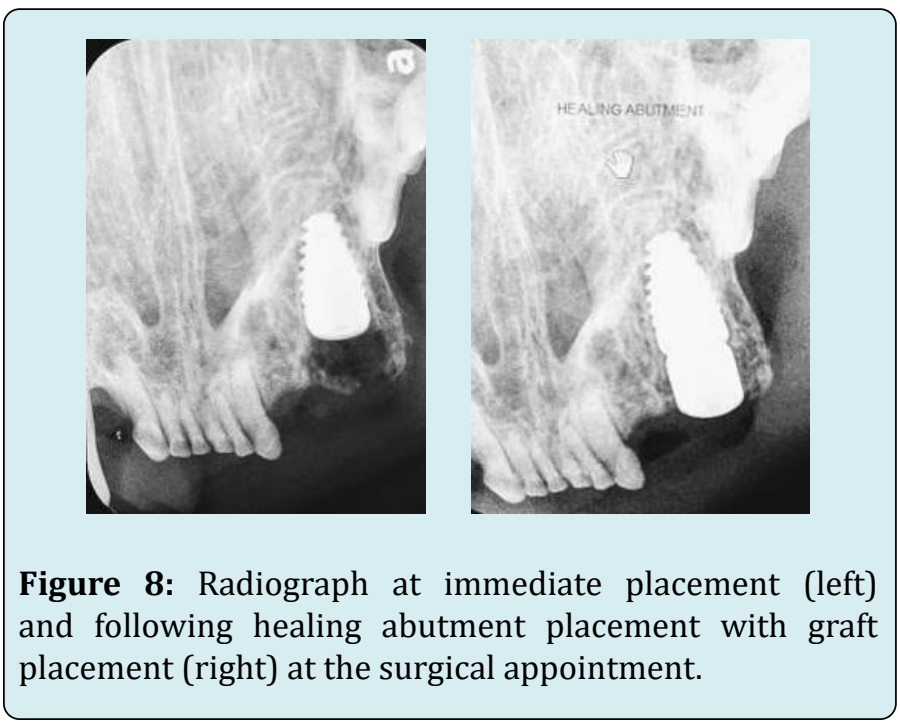

\section{Restorative Phase}

Because of the owner's busy schedule, re-evaluation was limited to e-mail photos of the surgical site at 3 weeks and 8 weeks post-operatively (Figure 9). The implant site continued to display minimal inflammation with no evidence of mucositis and/or peri implantitis.

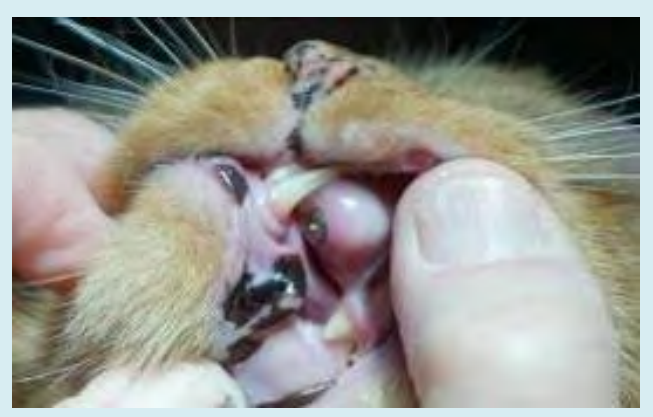

Figure 9: Clinical appearance at 8 week post-surgical demonstrating non-inflamed gingival tissue.

At 10 weeks post implant placement, the patient returned for intra-oral dental radiographs and a final Implant Stability Quotient (ISQ) value (Osstell USA, Columbia, Maryland) to assess osseointegration for a possible early restoration phase. The same feline general anesthetic protocol (GAP) for the surgical phase was repeated for the restorative phase. Dental radiographs were taken demonstrating excellent stability and osseointegration with an ISQ value of 74 recorded. ISQ value is an objective world standard for measuring implants stability.

The healing abutment (HA) was removed and a $5 \mathrm{~mm}$ diameter impression coping (OCO Biomedical) was attached to the implant (Figure 10). Rostral maxillary and mandibular impressions and bite registration were obtained with Vinyl Polysiloxane (VPS) (Benco Dental) The impression coping was then detached intraorally and replaced with a new $5 \mathrm{~mm}$ diameter HA repositioned into the implant to maintain the tissue emergence profile (Figure 11) and a final radiograph was taken (Figure12).

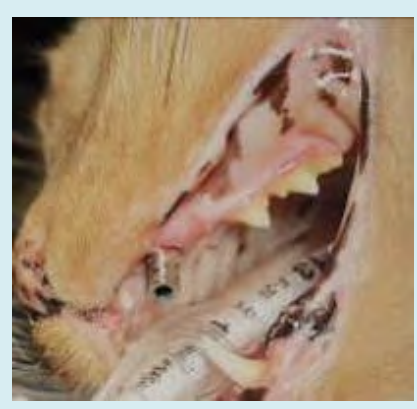

Figure 10: Impression coping placed into the implant at the restorative phase following removal of the healing abutment. 


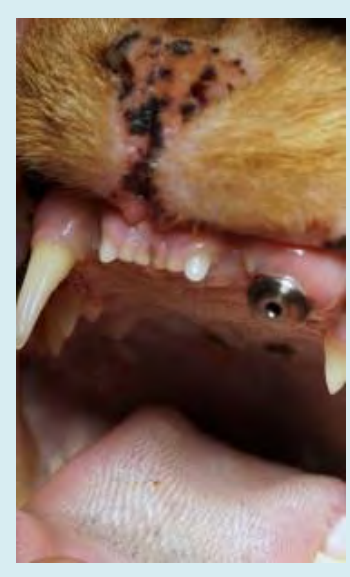

Figure 11: Clinical appearance at 10 weeks post-surgical demonstrating healthy non-inflamed gingival tissue around the healing abutment.

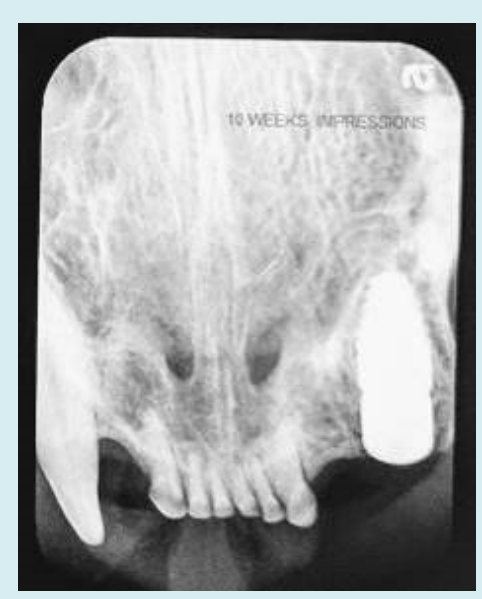

Figure 12: Radiograph at 10 weeks post-surgical demonstrating incorporation of the graft placed with the patients bone and lack of radiolucency between the implant and socket walls.

Maintenance of healthy thick keratinized tissue (KT) around the implant is crucial for long term implant and bone stability. KT minimizes bone resorption and inflammation around the prosthetics under function $[17,18]$.

\section{Customized Digital Workflow for Veterinary Prosthetics}

The impressions were sent to the dental lab (Dental Prosthetics, Tucson, AZ) and a stone model was created with an implant analog within the model and scanned utilizing a 3 Shape D2000 scanner (3 Shape Inc., Warren, N)) to create a virtual model. Final components were designed incorporating 3 Shape software and CAD CAM milled (Figures 13).

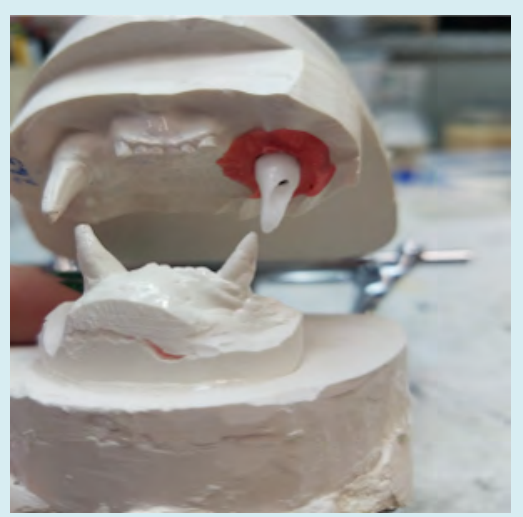

Figure 13: Cad/Cam milled wax crown on the mounted models to verify relationship with the opposing arch.

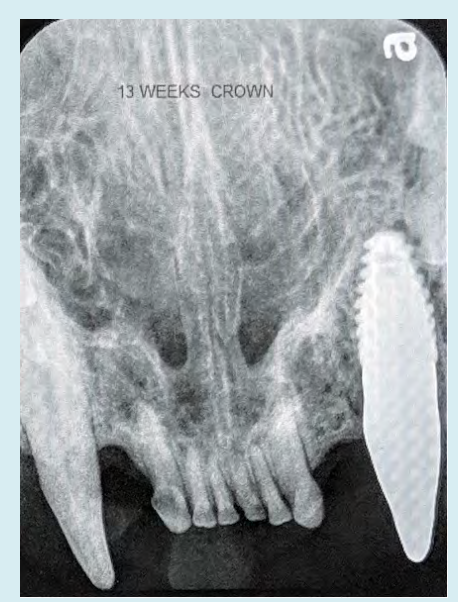

Figure 14: Radiograph at 13 weeks post-surgery with the screw retained restoration fixated to the implant taken to verify complete mating of the restoration and implant at the platform.

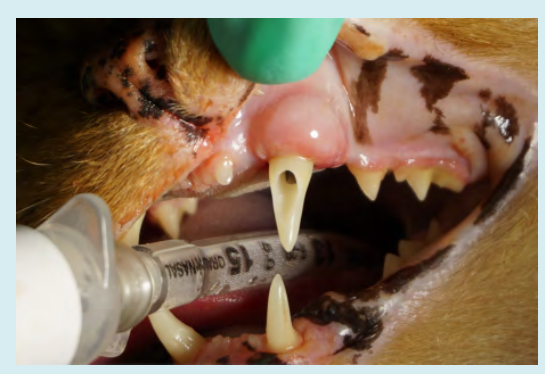

Figure 15: The screw retained restoration intraorally following torque of the fixation screw to secure the restoration to the implant.

Three weeks after dental impressions were taken, the patient returned for delivery of the final prosthetic components, a screw-retained zirconia crown on a custom 
titanium abutment. The restoration arrived from the laboratory as a one piece restoration with the zirconia crown luted to the titanium abutment that would be screw retained to the implant intraorally. The patient was again anesthetized following the general anesthetic protocol followed in the two previous procedures. An oral exam and intra oral radiographs were obtained to evaluate the peri-implant soft tissue and the quality of the bone surrounding the implant (Figure 14). The screw retained crown was soaked in a 0.12 \% Chlorhexidine Gluconate rinse (Delta Hex Oral Rinse w ZN, MWI Veterinary Products) for 2 minutes to prevent introduction of oral bacteria into the implant connection during restoration insertion. The restorations external hex connector was lined up with the implant platforms internal hex connector to get the correct rotational position and seated into the implant. The restorative fixation screw was inserted into the screw access channel and tightened to 25 Ncm following the manufactures recommendations and verified with a calibrated torque wrench (Figure 15). A piece of Teflon tape was placed into the crowns screw access channel and then the area over the tape was filled with a lightcured radiopaque, methacrylate-based flowable composite, PermaFlo (Ultradent Products,Inc. South Jordan, UT) to seal trhe screw access channel and match the surrounding restorations contours (Figures 16).

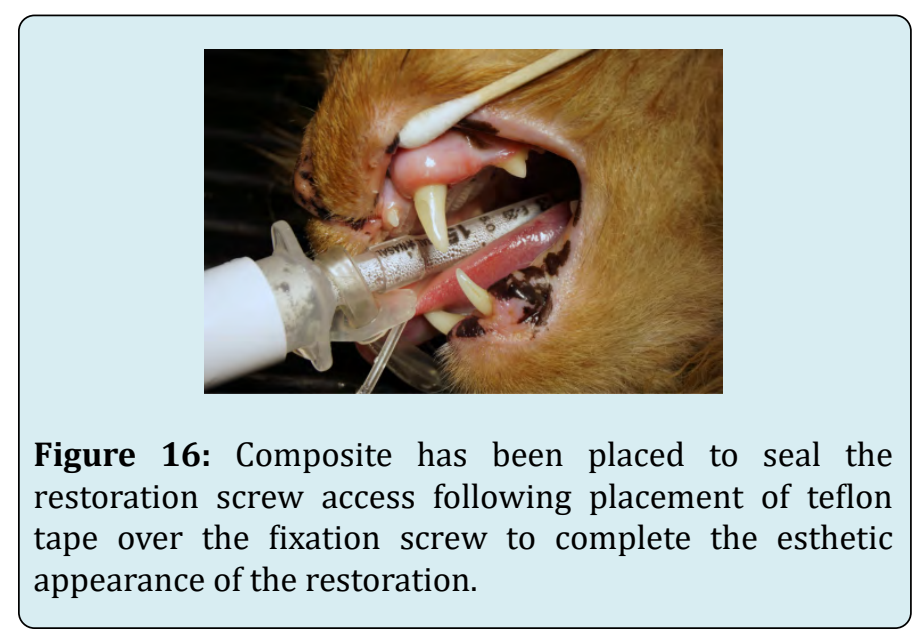

The nine day and six week re-call appointments presented with minimal inflammation and good implant stability related to the emergence profile of the restoration. The axial contour of the natural tooth or prosthetic crown as it relates to the adjacent soft tissue, as a good emergence profile has been shown improve the effectiveness of oral hygiene near the gingival sulcus limiting potential for soft tissue recession and marginal inflammation related to food impaction during mastication is eliminated [19] (Figures 17). Design of the restoration allows long-term maintenance of the gingival health without inflammation.

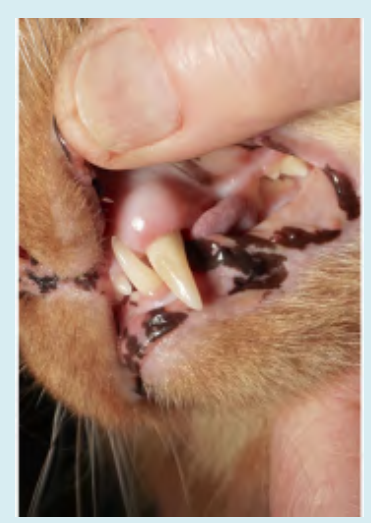

Figure 17: Clinical appearance 9 days post insertion of the implant restoration demonstrating non-inflamed gingival tissue.

At a four-year routine appointment, the maxillary canine implant/restoration demonstrated excellent tissue and bone stability (Figure 18). Minimal gingival inflammation was noted with a lack of bone loss even though the feline patient does not maintain daily oral hygiene that the human patient would practice.

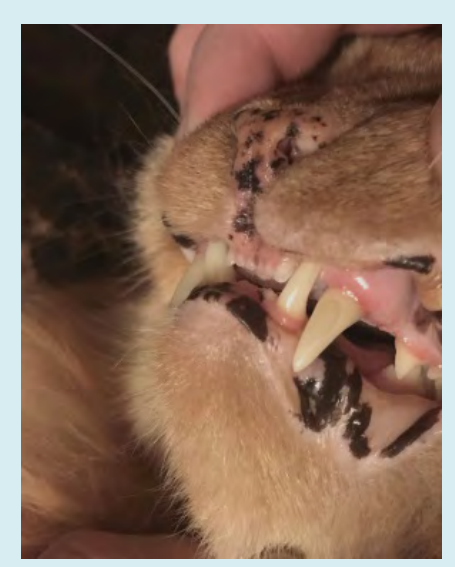

Figure 18: Four year routine follow-up demonstrating gingival health surrounding the implant and its restoration and an absence of gingival inflammation.

\section{Discussion}

Dental implants in the view of many veterinarians, under no circumstances, should ever be placed in dogs and felines for many reasons including lack of formal training programs [20]. The authors would agree that formal and rigorous training is necessary to develop acceptable and predictable outcomes. In contrast other disciplines that are universally accepted, such as, endodontics, orthodontics, and periodontics, are continually utilized to save, not extract, functional teeth in our pets. Ironically, the favored argument 


\section{Open Access Journal of Veterinary Science \& Research}

is that pets do very well without their teeth.

Many years ago there was very little evidence that endodontic and orthodontic treatment was a viable and predictable option for companion animals. Yet, over the years and with a number of published case reports, it has been accepted as a predictable valuable service that can be offered to pet owners [21-25]. Until recently there has been minimal evidence in the literature that dental implants have ever been applied in specialized treatment planning in canine and feline dentistry. Because clinical case documentation and short and long term follow up is lacking, many would consider this discipline experimental. However, the principal author has placed 60 implants in 40 feline patients with more than half being restored. All cases have complete documentation with many appearing in multiple publications in Veterinary and Human dental journals [26-28].

Feline Alveolar Osteitis is a common presenting problem that the veterinary dental clinician has to deal with routinely. Most cases require surgical extraction of the affected tooth, buccal bone osteoplasty, and extensive debridement before a tension free gingival flap is closed. In this case presentation we explore a paradigm shift in the treatment of AO with surgical extraction, utilizing the alveolar inflammatory changes (bone buttressing) and a osteotomy drilling protocol (osseodensification) to develop an ideal site for immediate implant placement, excellent primary stability and a prosthetic crown restoration 13 weeks following implant placement. This new treatment protocol has proven effective and predictable in over 40 plus cases. Although, implants may be viewed as being an esthetic solution, quality of life is important and has been shown to improve the feline's lifespan by improved eating ability and lip support. The implant and restoration not only provides a normal functioning maxillary canine yet prevents a common complication, lip entrapment, especially in the feline patient.

Customized digital planning is now being utilized in all our implant crown restoration cases compared to older techniques utlizing direct wax-ups. With digital planning, the dental laboratory creates a soft tissue model and subsequent castings employing advanced computer-aided design. The computer aided process produces more precise restorative results than the traditional methods of the past.

Detailed post-op instructions for the long-term survival of the implant/crown restoration are discussed and sent home with the owner in the departing instructions. Softer food only (no dry food), no toys and gentle cleaning of the prosthesis with soft moist swabs daily for 2-4 weeks following implant placement. Pain medication and antibiotics are dispensed on case by case basis.

\section{Conclusion}

Utilizing AO inflammatory socket modifications combined with an innovative osteotomy technique (osseodensification) the surgical site can be optimized by maintenance of the surrounding bone and its quality and implant stability thus preventing future collapse of the buccal plate. Immediate implant /restoration can be a manageable approach to restore the feline patient to full function and preclude the serious common complication of feline maxillary canine extraction, lip-entrapment. Extraction and immediate implant placement with early loading utilizing the new osteotomy protocol with Densah burs provides a predictable treatment for this common feline dental condition.

\section{References}

1. Lewis JR, Okuda A, Shofer FS, Pachtinger G, Harvey CE, et al. (2008) Significant association between tooth extrusion and tooth resorption in domestic cats. J Vet Dent 25 (2): 86-95.

2. Newman MG, Takei HH, Klokkevold PR (2015) Carranza's Clinical Periodontology, 12th (Edn.), St. Louis Missouri: Sanders pp: 875

3. Beebe DE, Gengler WR (2007) Osseous surgery to augment treatment of chronic periodontitis of canine teeth in a cat. J Vet Dent 24(1): 30-38.

4. D’Astous J (2015) Periodontology: An overview of alveolar bone expansion. Can Vet J Mar 56(3): 295-300.

5. Bell CM, Soukup JW (2015) Histological, Clinical, and Radiographic Findings of Alveolar bone expansion and osteomyelitis in the jaws of cats. Veterinary Pathology 52(5): 910-918.

6. Huwais S, Meyer EG (2016) A Novel Osseous Densification approach in Implant Osteotomy Preparation to increase Biomechanical Primary Stability, Bone Mineral Density, and Bone-to- Implant contact. J Oral Maxillofac Implants 32(1): 27-36.

7. Summers KB (1994) A New concept in Maxillary implant surgery: The osteotome technique. Compendium 15(2): 152-162.

8. Green JR, Nemzek JA, Arnoczky SP, Johnson LL, Balas MS (1994) The effect of bone composition on early fixation of porous-coated implants. J Arthroplasty 14(1): 91-97.

9. Kold S, Rihbek O, Vester M, Overgaard S, Soballe K (2005) Bone compaction enhances fixation of weight bearing titanium implants. Clin orthop Relat Res 431: 138-144. 


\section{Open Access Journal of Veterinary Science \& Research}

10. Trisi P, Bernardini M, Falco A, Podaliri M (2016) New Osseodensification implant site preparation method to increase bone density in low-density bone. In Vitro evaluation in sheep. Implant Dentistry 25(1): 24-31.

11. Perren SM, Huggler A, Russenberger M (1969) The reaction of cortical bone to compression. ACTA Orthop Scand Suppl 125: 19-29.

12. Trisi P, Todisco M, Comsolo U, Travaglini D (2011) High versus low implant torque: A histological, histomorphometric, and bio medical study in sheep mandible. Int J Oral Maxillofac Implants 26(4): 837-849.

13. Huwais S, Meyer EG (2017) A Novel osseous densification approaching implant osteotomy preparation to increase bio mechanical primary stability, bone mineral density and bone-to- implant contact. Int J Oral Max-illofac Implants 32(1): 27-36.

14. Rodrigo D, Aracil L, Martin C, Sans M (2010) Diagnosis of implant stability and its impact on implant survival : A prospective case series study. Clin Oral Impl Res 21(3): 255-261.

15. Chu SJ, Salama MA, Salama H, Garber D, Saito H, et al. (2012) The dual-zone thera-peutic concept of managing immediate implant placement and provisional restoration in anterior extractionug. 33(7).

16. Baltayan S, Anfruns JP, Aghaloo T, Moy P (2016) The predictive value of resonance frequency analysis in surgical placement and loading endosseous implants. J Oral Maxillofac Surgery 74(6): 1145-1152.

17. Rieder C (1996) Customized implant abutment copings to achieve biologic mechanical and esthetic objectives. Int J Periodont Restorative Dent 16(1): 20-29.

18. Chiche FA, Leriche MA (1998) Multi-diciplinary implant dentistry for the improved aesthetics and function. Pract Periodontics Aesthetics Dent 10 (2): 177-186.

19. Croll BM (1990) Emergence profile in natural tooth contour. Part II: Clinical Considerations. J Prosthet Dent 63(4): 374-379.

20. Tannenbaum J, Arzi B, Reiter AM (2013) The case against the use of dental Implants in dogs and cats. J AM Vet Med Assoc 243(12): 1680- 1685.

21. Visser C (1990) Endodontology: Chronic Maxillary Sinus abcessation in the Canine. J Vet Dent 7(2): 12-13.

22. Eisner E (1990) Transcoronal approach for endodontic access to the fourth maxillary premolar in dogs. J Vet Dent 7(4): 22-23.

23. Willams C (1986) Endodontics. Vet Clin North Am Small Anim Pract 16(5): 875-893.

24. Visser C (1991) Coronal access of the canine dentition. J Vet Dent 8(4): 12-16.

25. Kuntsi-Vaattovaara H, Verstraete F, Kass P (2002) Results of root canal treatment in dogs 127 cases (1995-2000). J Am Vet Med Assoc 220(6): 775-780.

26. Mele RE, Kurtzman GM (2019) New paradigm shift in Feline Dental Implants in Maxillary Alveolar Osteitis treatment with osseodensification. Spectrum Implants Spring 11(3): 1-8.

27. Mele RE, Caiafa A, Kurtzman GM (2017) Feline Dental Implants: Long term follow - up of two cases. J Vet Dent 34 (4): 249-258.

28. Mele RE, Caiafa A, Kurtzman GM (2016) Dental Implants and Incisor Bridge Placement in a Dog. J Vet Dent 33(4): 249-258. 